\title{
Self-organized nanostructured anodic oxides for display applications
}

\author{
P. Jaguiro, A. Stsiapanau, A. Hubarevich, Y. Mukha and A. Smirnov \\ Belarusian State University of Informatics and Radioelectronics, \\ Laboratory of Information Displays, \\ 6, P. Brovki str., 220013 Minsk, Belarus
}

\begin{abstract}
Electrochemical technologies have a high potential for display applications because of their cheapness and simplicity, easiness to scaling to large substrates and lowtemperature nature. However, in major display technologies the oxide films should be deposited on transparent conductive substrate, usually ITO on glass. For dielectric substrates like glasses, a special technology of current control is applied to anodizing metal films, which changes the oxide porous structure in a final stage and prevents formation of metal islands. To transform the residual metal nanowires into oxide, a special fading process similar to anoding bonding can be done. Usually, high reactivity electrolytes are used in the anodizing process, which destroys ITO layers. We have analyzed chemical properties of ITO in various anodizing electrolytes and found some suitable reagents and compositions. A lot of functional layers can be created by anodizing. For example, different filters may be formed by filling the pores by ink jet printing. Porous oxides can have low refractive indexes - lower than any bulk material, and can be used as effective antireflective coatings. A titanium oxide cover film forms "self-cleaning" surface due to its semiconductor photonics properties and oxygen production.
\end{abstract}

Keywords: transparent and functional layers, displays, photonics, electrochemical anodizing.

Manuscript received 18.12.09; accepted for publication 08.07.10; published online 30.09.10.

\section{Introduction}

Electrochemical technologies have a high potential for displays and photonics applications because of its cheapness and simplicity, easiness to scaling to large substrates and low-temperature nature [1]. However, electrochemical processes at higher temperatures when glass substrates remain solid but get some ionic conductivity can provide a drastically wide range of possible applications.

Both displays and photonic devices operate with transparent substrates and layers, therefore, from the practical point of view, anodizing technologies for $\mathrm{Al}, \mathrm{Ti}$ and $\mathrm{W}$ foils and thin films can be interesting. The anodic oxides of metals are widely used in displays and photonics for vertical alignment of liquid crystals [2], polarizers [3], optical filters [4], self-cleaning glasses [5], sensors [6], photolysis and electrochromic devices based on valve metal oxide layers [7], fading created oxide layers and carrying out anodizing processes over transparent conductive films is a point of intense interest for researchers and engineers.
Some anodic nanoporous oxides like $\mathrm{TiO}_{2}$ and $\mathrm{WO}_{3}$ possess semiconductor properties that can be effectively used in displays and photovoltaic applications. However, processes of their formation and especially through anodizing over ITO layers are not sufficiently investigated and clear up to date. A primary goal of this work is to investigate foil to glass anodic bonding technology, because anodic bonding is well known [8] to provide excellent adhesion and vacuum sealing quality. However, to bond bulk metal, the glass with correspondent thermal expansion should be used. For $\mathrm{Al}$ correspondent glasses are absent at all, for $\mathrm{Ti}$ and W correspondent glasses get ionic conductivity only at rather high temperatures. But for the metal foils due to their high elasticity, it is possible to use metal-glass systems with a significant difference in thermal expansion [9]. Particularly, it is possible to use sodium glasses and aluminum foil [10]. Note that for Ti and W foils special surface treatment is needed. The process of foil to glass anodic bonding is illustrated in Fig. 1. For aluminum foil and sodium glass, the bonding process takes about 10 minutes at $270-300{ }^{\circ} \mathrm{C}$ and $\sim 5 \mathrm{~mA} / \mathrm{cm}^{2}$ 
current density provided with the negative 1200-1600 V bias at high voltage electrode. It is necessary to polish foil for the best adhesion. However, polishing is not enough to achieve well organized honeycomb structure of anodic oxide (Fig. 2).

Thermal annealing of the foil is more important for the best pore organizing.

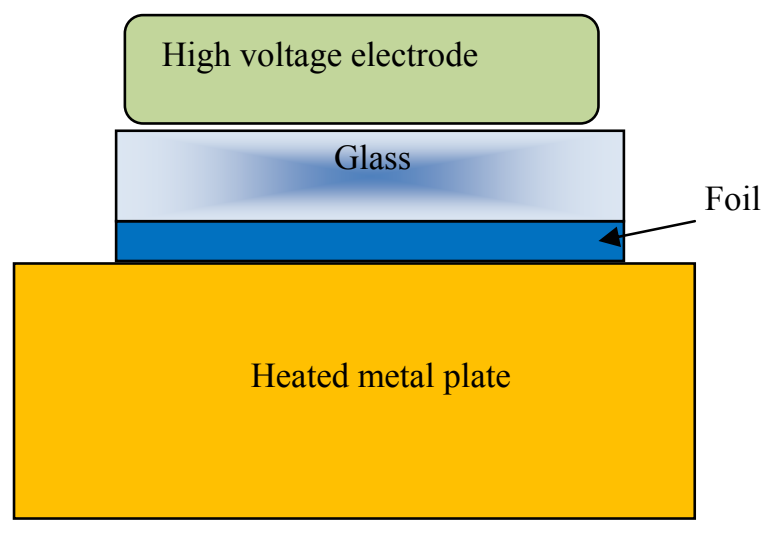

Fig. 1. Scheme of anodic bonding.

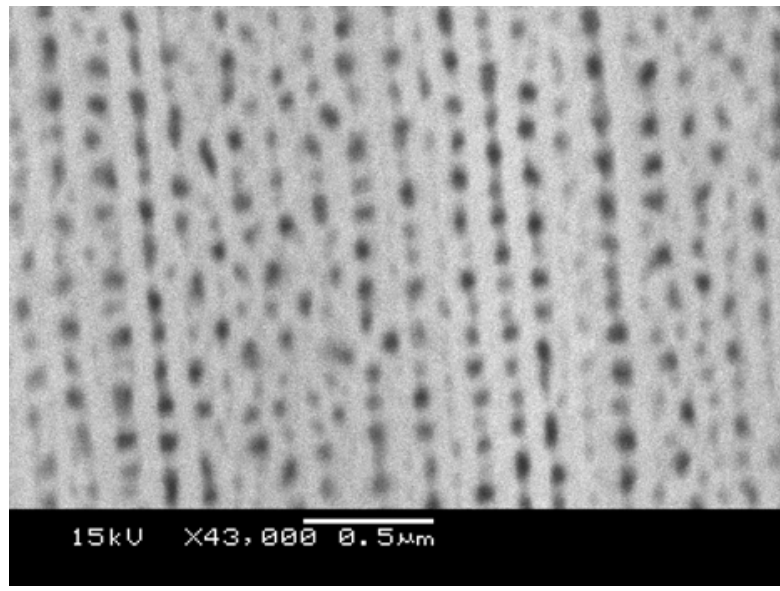

Fig. 2. Anodized polished Al foil.

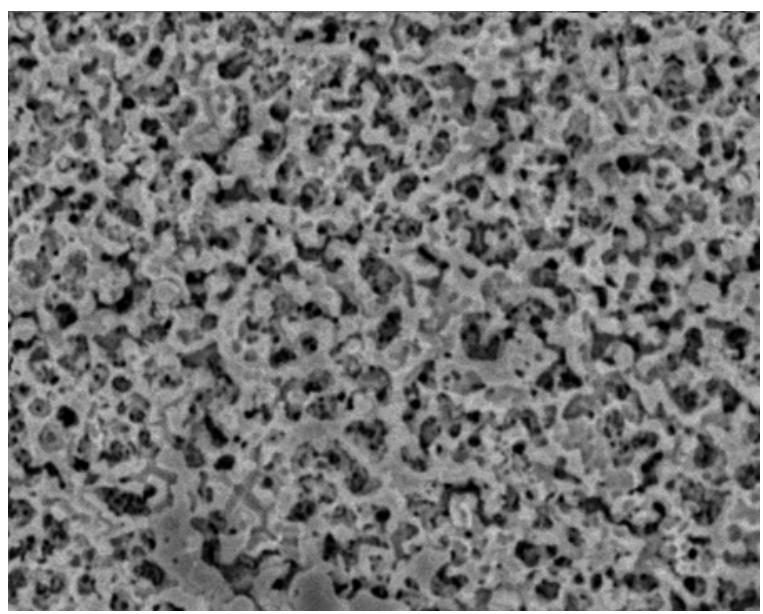

\section{Through anodizing the metal films on insulator substrates}

When anodizing foil, it appears that a lot of metal islands remains at the interface between isolated substrate and anodic oxide. The reason is local differences in the foil thickness and anodizing current. If islands are created, the anodizing process is stopped at the moment, because of current interruption. Special technology of current control is applied to anodizing metal films on insulator substrates, which changes the oxide porous structure in the final stage and prevents formation of metal islands. An additional factor that lowers transparency of the film is presence of many vertical metal nanowires in anodic oxides [11]. It is very good for polarizes, but bad for transparency. To transform the nanowires into oxide, a special fading process similar to anoding bonding can be done [12]. It is possible to create a lot of functional layers by filling the pores, for example, by ink jet printing [13]. Note that porous oxides can possess low refractive indexes - lower than those of any bulk materials, and can be used as effective antireflective coatings. Titanium oxide cover film forms "self-cleaning" surface due to its semiconductor photonics properties and oxygen production.

\section{Through anodizing the metal films on transparent conductive substrates}

For display technologies and photonics, the oxide films should be deposited on transparent conductive substrate, usually ITO on glass. However, high reactivity electrolytes are used in the anodizing process, which destroys ITO layers. We have analyzed chemical properties of ITO in different anodizing electrolytes and found some suitable reagents and compositions. Mechanisms of ITO passivation have been discussed in details. Current voltage characteristics of anodic ITO dissolution have been presented and analyzed. Overheating and gas evolution as destroying factors have been under consideration, too.

In Fig. 3, nanosponge structure of $\mathrm{WO}_{3}$ anodized in ITO compatible electrolyte is presented.

\section{Porous alumina template masking}

It is possible to achieve many useful structures by anodizing the bi-layer structures, where top porous anodic oxide is used as a template for anodizing or etching the bottom layer. For example, titania columnar structure [1] and ultra-black silicon can be formed [14]. Masking makes easier the pore nucleation process in the bottom layer, therefore more regular porous structures in less aggressive electrolytes can be formed in the bottom layer.

Fig. 3. Nanosponge structure of $\mathrm{WO}_{3}$. 


\section{Transparent conductive aluminum nanomesh}

It is possible to form a transparent conductive aluminum nanomesh on glass substrate from thin aluminum layer by simple anodizing process. The anodizing parameters are changed in the final stage of the process [15], which allows to open transparent windows and leave a nanomesh from aluminum residues (see Fig. 4).

SEM photo of the aluminum nanomesh after selective etching the $\mathrm{Al}_{2} \mathrm{O}_{3}$ skeleton is presented in Fig. 5.

In the nanomesh, transparent and conductive functions are separated in space, but this feature doesn't come out due to a sub-wavelength size of the nanomesh pitch. It allows overcoming some fundamental limitations that are specific to ITO and ultra-thin metal films, where conduction electrons operate as electric charge carriers and light absorbers simultaneously.

In Fig. 6, geometrical structure of the nanomesh and correspondent theoretical results (resistance and transmittance for different pitch values) are presented.

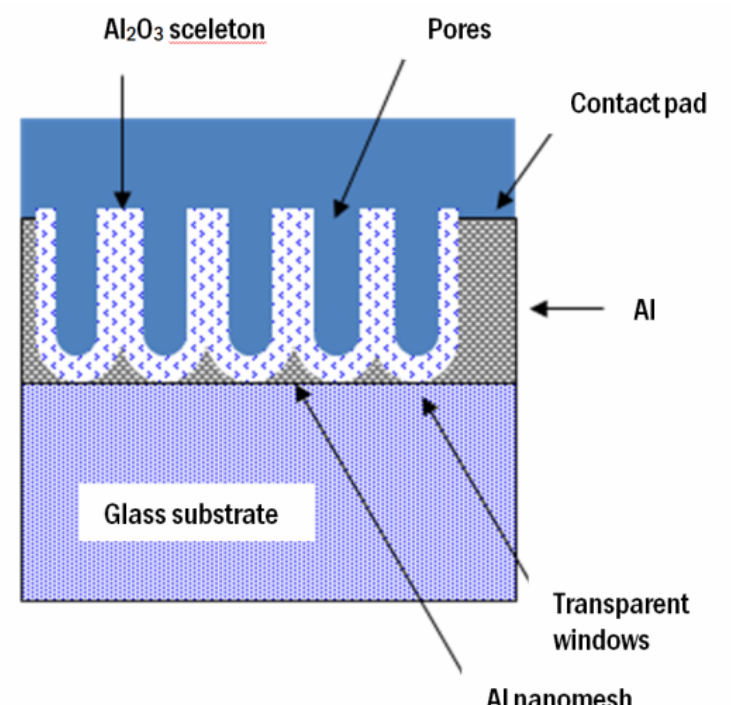

Fig. 4. Structure of the transparent conductive nanomesh.

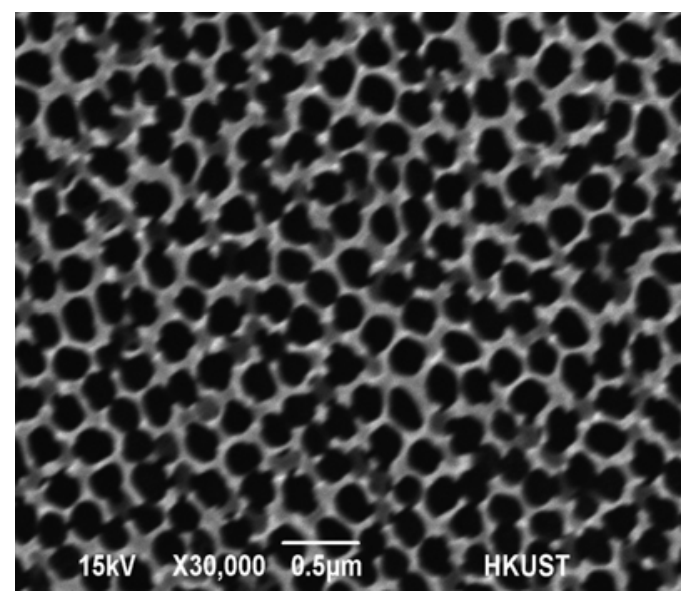

Fig. 5. SEM photo of the aluminum nanomesh.
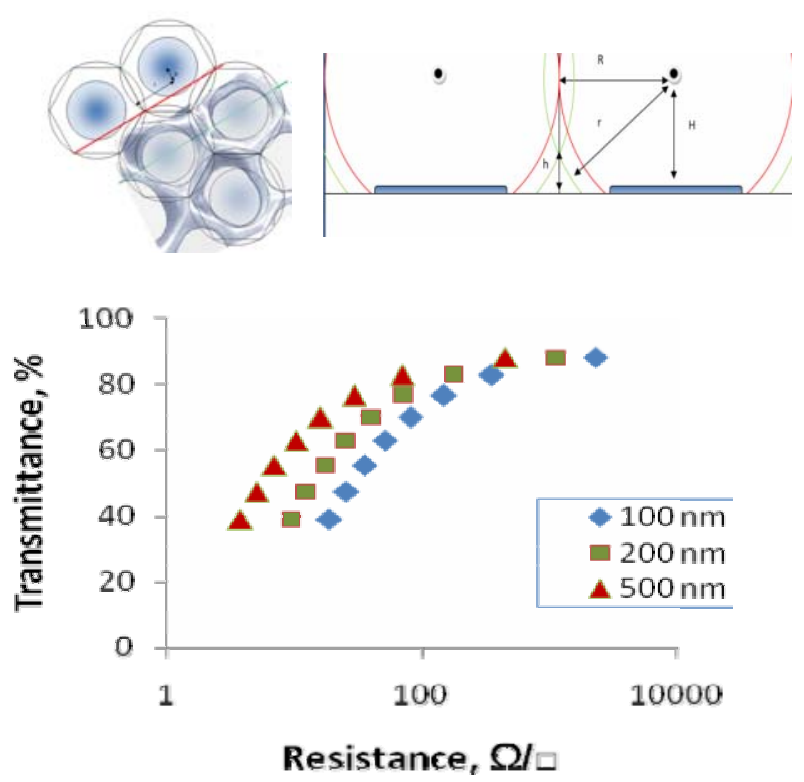

Fig. 6. Geometrical structure and correspondent theoretical calculations for the nanomesh.

\section{Conclusion}

Electrochemistry based technologies to fabricate transparent porous anodic alumina layers and aluminum nanomesh, which can work as alignment layers, color filters, light absorbers and transparent conductive films have been proposed. These technologies are simple, cheap, low-temperature and scalable to large substrates and roll-to-roll techniques.

\section{References}

1.S. Lazarouk, P. Jaguiro, V. Labunov, D. Sasinovich, A. Smirnov, A. Muravski, V. Chigrinov and H. Kwok, Anodizing technique for liquid crystal displays // SID Eurodisplay, Moscow, 2007.

2. T. Maeda and K. Hiroshima, Vertically aligned nematic liquid crystal on anodic porous alumina // Jpn. J. Appl. Phys. 43(8A), p. 1004-1006 (2004).

3. L. Huang, M. Saito and M. Miyagi, Polarization characteristics of alumina films anodized at low temperature // Jpn. J. Appl. Ph ys. 32(7), p. 3169-3174 (1993).

4. V. Kochergin and H. Foell, Novel optical elements made from porous Si // Mater. Sci. and Eng.: R: Repts 52(4-6), p. 93-140 (2006).

5. A. Fujishima, X. Zhang and D. Tryk, $\mathrm{TiO}_{2}$ photocatalysis and related surface phenomena // Surf. Sci. Repts 63(12), p. 515-582 (2008).

6. K. Mor, O. Varghesea, M. Paulosec, K. Ongc and C. Grimes, Fabrication of hydrogen sensors with transparent titanium oxide nanotube-array thin films as sensing elements // Thin Solid Films 496(1), p. 42-48 (2006). 
7. C. Granqvist, Oxide electrochromics: Why, how, and whither // Solar Energy Materials and Solar Cells 92(2), p. 203-208 (2008).

8. K. Knowles and V. Helvoort, Anodic bonding // Intern. Mater. Rev. 51(5), p. 273-311 (2006).

9. S. Park and M. Kim, Fabrication method of spacers with high aspect ratio - Used in a field emission display (FED) // Microsystem Technologies 7(1), p. 3235 (2001).

10. S. Lee, M. Lee, W. Choi, D. Lee and Y. Kim, Characteristics of ACPDP test panels with aluminum fence electrode formed via anodic bonding with sodalime glass // J. SID 16/12, p. 1219-1227 (2008).

11. M. Saito, Y. Shiga and M. Miyagi, Unoxidized aluminum particles in anodic alumina films // $\mathrm{J}$. Electrochem. Soc. 140 (7), p. 1907-1911 (1993).

12. S. Park, H. Lee, J. Cho and K. Lee, Nanoporous anodic alumina film on glass: improving transparency by an ion-drift process // Electrochem. and Solid-state Lett. 8(3), p. 5-7 (2005).
13. M. Ikeda, T. Abe and K. Matsuo, Image forming method, process for producing decorative aluminum plate, apparatus for carrying out the process, decorative aluminum plate, and recording medium, USA Patent No. 5,786,835 (1998).

14. H. Sai, H. Fujii, K. Arafune, Y. Ohshita and M. Yamaguchi, Antireflective subwavelength structures on crystalline Si fabricated using directly formed anodic porous alumina masks // Appl. Phys. Lett. 88 (20), 201116 (2006).

15. A. Stsiapanau, P. Jaguiro, A. Smirnov, H. Kwok, A. Murauski and Y. Jacob, Nanostructured metal transparent conductive layer and method of its self-ordered fabrication from valve metal film, USA Patent No. application 61/213,283 on 05/26/2009 (2009). 